\title{
Antropología
}

\section{Atender a los pacientes con trastornos mentales desde la perspectiva de la enfermera de atención primaria}

\section{Care for patients with mental disorders from the perspective of the primary care nurse}

\section{Cuidado ao portador de transtorno mental sob a ótica do enfermei- ro da atenção básica}

Dielson Alves de Sousa ${ }^{1}$; Amanda Lúcia Barreto Dantas ${ }^{2}$; Laurimary Caminha Veloso ${ }^{2}$; Ana Maria Menezes Neiva Eulálio Amorim ${ }^{3}$

${ }^{1}$ Acadêmico de Enfermagem da Faculdade Santo Agostinho - FSA. Teresina - PI. ${ }^{2}$ Enfermeira Mestre em Enfermagem e Docente da FSA. ${ }^{3}$ Psicóloga Mestre em Psicologia e Docente da FSA.

Cómo citar este artículo en edición digital: Alves de Sousa, D; Barreto Dantas, A.L; Caminha Veloso, L; Eulálio Amorim, A.Ma .M. (2013) Atender a los pacientes con trastornos mentales desde la perspectiva de la enfermera de atención primaria. Cultura de los Cuidados.17, 37. Disponible en: <http://dx.doi.org/10.7184/cuid.2013.37.07>

Correspondência: Dielson Alves de Sousa Quadra H, Casa 40, Residencial Taquari, Bairro Vale Quem Tem, Teresina, Piauí, Brasil. CEP 64057-020. Correo electrónico: dielson_alves@hotmail.com. Tel. (086) 9446-0209 / (086) 3235-1168

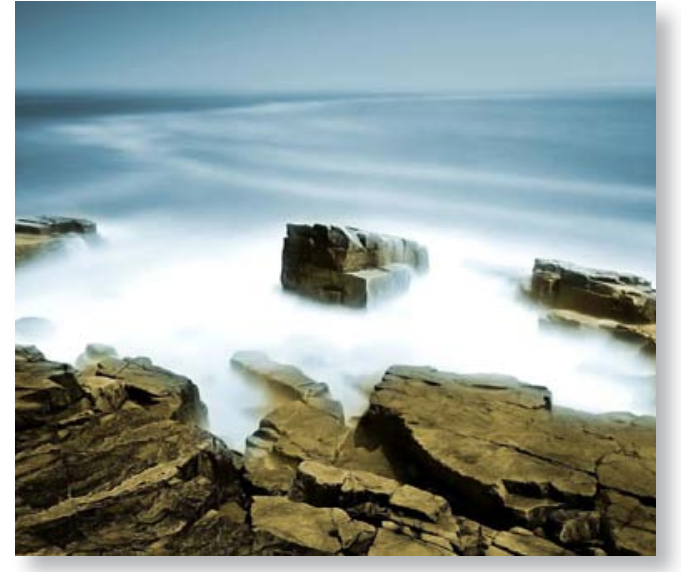

ABSTRACT

One in four people will be affected by a mental disorder at some stage in their life, regardless of socioeconomic class, causing considerable impact on their social environment. The nurse as an integral member of the FHT is characterized as a social agent participant multidisciplinary team linked to various health practices, including mental health co- llective and individual. This is a qualitative, exploratory and descriptive in order to identify and analyze the perception of nurses of primary care front bearer of mental disorder. Were conducted semi-structured interviews with eight nurses in ESF in the Central/North Teresina/PI who agreed to participate. The data were analyzed according to thematic analysis Minayo and grouped into three distinct categories: Stigma related Bearer of Mental Disorder; care Bearer Shares of Mental Disorder in Primary Care; Need for coordination of services. At this point it is necessary to delineate the role of each individual member of society. Perhaps the point where there is a component of subjective distress associated with any disease. Seeking to maintain a culture of care that incorporates new concepts pertaining to mental illness.

Keywords: Mental Health. Nursing. Care. Primary Care. 


\section{RESUMEN}

Una de cada cuatro personas se verán afectadas por un trastorno mental en algún momento de su vida, sin importar la clase socioeconómica, causando gran impacto en su entorno social. La enfermera como miembro integrante de la ESF se caracteriza por ser un equipo de agentes sociales participantes multidisciplinaria vinculada a las prácticas de salud diferentes, incluyendo la salud mental colectiva e individual. Esta es una orden cualitativo, exploratorio y descriptivo para identificar y analizar la percepción de las enfermeras de atención frente al portador primario de trastorno mental. Se llevaron a cabo entrevistas semi-estructuradas con ocho enfermeras en el FSE en Teresina Centro / Norte / PI que aceptaron participar. Los datos fueron analizados de acuerdo a la temática Minayo análisis y agrupados en tres categorías distintas: el estigma relacionado al portador de trastorno mental, las acciones de cuidado al portador de trastorno mental en la atención primaria; Necesidad de coordinación de los servicios. En este punto, es necesario definir el papel de cada miembro de la sociedad. Tal vez el punto en el que hay un componente de angustia subjetiva asociado con ninguna enfermedad. Tratando de mantener una cultura de atención que incorpora nuevos conceptos relacionados con la enfermedad mental.

Palabras clave: Salud Mental. Enfermería. Cuidados. Atención Primaria.

\section{RESUMO}

Uma em cada quatro pessoas será afetada por um transtorno mental em dada fase da sua vida, independentemente de classe socioeconômica, causando considerável impacto em seu meio social. O enfermeiro enquanto membro integrante da ESF caracteriza-se como agente social participante do trabalho da equipe multiprofissional vinculado às diversas práticas de saúde, incluindo a saúde mental coletiva e individual. Trata-se de uma pesquisa qualitativa, exploratória e descritiva com o objetivo de identificar e analisar a percepção do enfermeiro da atenção básica frente o cuidado ao portador de transtorno mental. Foram realizadas entrevistas semi-estruturadas com 8 enfermeiros atuantes em ESF na Região Centro/Norte de Teresina/PI que aceitaram participar da pesquisa. Os dados foram analisados conforme análise temática segundo Minayo e agrupados em três categorias distintas: Estigma relacionado ao Portador de Transtorno Mental; Ações de cuidado ao Portador de Transtorno Mental na Atenção Básica; Necessidade de articulação dos serviços. Neste momento é necessário delinear o papel de cada sujeito integrante da sociedade civil. Quem sabe do ponto em que existe um componente de sofrimento subjetivo associado a toda e qualquer doença. Buscando manter uma cultura de cuidados que incorpore novos conceitos pertencentes ao adoecimento mental.

Palavras-chave: Saúde Mental. Enfermagem. Cuidado. Atenção Básica.

\section{INTRODUÇÃO}

É sabido que o Brasil realiza, há pelo menos 30 anos, profundas mudanças na assistência pública em saúde mental. A atual Política Nacional de Saúde Mental do Sistema Único de Saúde (SUS), apoiada na lei 10.216/01 e fundamentada na Declaração de Caracas, busca consolidar um modelo de atenção à saúde mental aberto e de base comunitária, garantindo assim a livre circulação das pessoas com transtornos mentais pelos serviços, comunidade e cidade, e oferece cuidados com base nos recursos que a comunidade possui. Ainda, tem como diretriz principal a redução gradual e 
planejada de leitos em hospitais psiquiátricos, com a desinstitucionalização de pessoas com longo histórico de internações (Brasil, 2005; Brasil, 2008a).

Esse paradigma passa a ganhar um novo contorno institucional: os Centros de Atenção Psicossocial (CAPS). Os CAPS poderão constituir-se, segundo a Portaria n. ${ }^{\circ}$ 336/GM, de 19 de fevereiro de 2002, nas seguintes modalidades de serviços: CAPS I, CAPS II e CAPS III, definidos por ordem crescente de porte/ complexidade e abrangência populacional, e deverão estar capacitados para realizar prioritariamente o atendimento de pacientes com transtornos mentais severos e persistentes em sua área territorial, em regime de tratamento intensivo, semi-intensivo e não-intensivo (Brasil, 2002; Gama, 2008; Santos, 2008; Chamma et. al., 2011)

Vários outros serviços e programas de saúde mental se organizaram e buscam consolidar seu papel como de fundamental importância na reestruturação da rede assistencial brasileira em substituição aos hospitais psiquiátricos. São eles os centros de convivência e cultura, os serviços residenciais terapêuticos, os hospitais gerais, os ambulatórios de saúde mental, a integração da rede básica com os centros de atenção diária e o programa de reinserção comunitária por meio de bolsas-auxílio (Brasil, 2003; Brasil, 2005; Moura, 2010).

Para Souza et. al., (2007) esse cenário se apresenta como um grande desafio aos pesquisadores, gestores, órgãos e instituições formadoras e usuários do serviço de saúde, cabendo-lhes, portanto, de forma democrática e pactuada, articular saberes e desenvolver estratégias de mudanças no modelo assistencial e na legislação em saúde mental, para que, gradativamente, observem-se mudanças culturais secularmente enraizadas na sociedade.

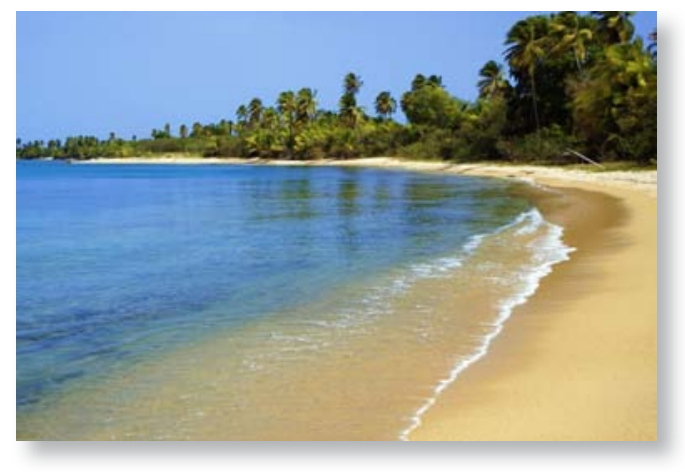

Nessa perspectiva, Townsend (2002) e Stefanelli (2011), compreendem a saúde mental como o estado de funcionamento harmônico, uma adaptação bem sucedida de estresse do ambiente interno ou externo, evidenciada por pensamentos, sentimentos e comportamentos que são apropriados para a idade e equivalentes com normas locais e culturais, sendo as pessoas capazes de reconhecer suas limitações. Da mesma forma caracterizam a doença mental por resposta desajustada aos fatores referenciados que interferem no funcionamento social, ocupacional e/ou físico do individuo (Townsend, 2002; Stefanelli, 2011).

Por outro lado, Amarante (2007) e o Ministério da Saúde (Brasil, 2009b) afirmam que conceituar saúde e doença mental é bastante complexo, plural, intersetorial, com transversalidades de saberes, uma vez que perpassa as dimensões psicossociais do processo saúde-doença dos sujeitos e do coletivo.

Em 1994 era proposto pelo Ministério da Saúde o Programa Saúde da Família (PSF), vertente brasileira da Atenção Básica à Saúde, na intenção de servir como instrumento de reorganização do Sistema Único de Saúde, substituindo o modelo hospitalocêntrico por uma prática de base comunitária com acesso igualitário e municipalizado (Leal, 2010; Moura, 2010).

A Atenção Primária à Saúde (APS) é vista como uma das principais estratégias para 
priorização das ações de saúde mental, uma vez que se caracteriza como porta de entrada para o sistema de saúde vigente em nosso país e representa um complexo conjunto de conhecimentos e procedimentos e demanda uma intervenção ampla em diversos aspectos. Assim possibilitando ampliar o acesso ao cuidado em saúde mental em âmbito comunitário e familiar (Brasil, 2009a).

O Ministério da Saúde aos 28 dias do mês de março de 2006 aprovou a Política Nacional de Atenção Básica (PNAB), estabelecendo a revisão de diretrizes e normas para a organização da Atenção Básica para a estratégia SF e o Programa Agentes Comunitários de Saúde (PACS) (Brasil, 2006).

As discussões que possibilitaram alcançar o formato final da PNAB fundamentaram-se nos eixos transversais dos princípios assistenciais e organizativos do Sistema Único de Saúde (SUS), em um processo que agregou atores diversos nos municípios, nos estados e na federação (Brasil, 2007a).

Mendes (2009) e Stefanelli (2011) afirmam que, segundo estimativas da Organização Mundial da Saúde (OMS), uma em cada quatro pessoas será afetada por um transtorno mental em dada fase da sua vida, em um percentual de $25 \%$ da população, afetando pessoas de todos os países e de diferentes idades e sexo, independentemente de classe socioeconômica, e causando considerável impacto no indivíduo, na família e na sociedade.

O profissional de enfermagem como membro integrante da Equipe de Saúde da Família (ESF), pode ser também caracterizado como agente social participante do trabalho da equipe multiprofissional e está vinculado às diversas práticas de saúde, incluindo a assistência à saúde mental coletiva e individual (Mendes, 2009; Brasil, 2011a).
O trabalho de enfermagem em USB como prática social é um dos meios de produção de saúde mental que visam a fazer a busca ativa da pessoa acometida por esse agravo, envolvendo a família no processo de reabilitação e reinserção do indivíduo com doença mental revelando as influências que sofre e as tendências das quais que incorpora para atender à nova política de saúde mental: a inclusão social (Brasil, 2006; Moura, 2010).

Segundo dados do Ministério da Saúde (Brasil, 2010a) o acesso à atenção em saúde mental alargou, chegando a 66\% de cobertura nacional, com forte participação da atenção básica e de ações intersetoriais. O país conta 1.620 CAPS, no qual a região nordeste apresenta o maior número, mesmo possuindo a segunda maior densidade demográfica brasileira, conta com um total de 597, destes 38 encontram-se funcionantes no Piauí.

A rede de serviços em saúde mental na capital piauiense, Teresina, hoje, conta com 07 CAPS, sendo: 04 CAPS II, 01 CAPS III, 01 CAPS ad e 01 CAPS i, distribuídos territorialmente; 01 Hospital Dia; 03 Residências Terapêuticas; 01 hospital psiquiátrico (Lopes, 2007; Brasil, 2010a; Brasil, 2011a).

A Atenção Básica no município é composta pelo PSF, implantado em outubro de 1997, dispondo de uma cobertura atual de $86,1 \%$ da população ao que se refere à assistência prestada pelas ESF; cada equipe atende 1.500 famílias, aproximadamente. Estas equipes são distribuídas em 03 regionais de saúde: Sul, Centro-Norte e Leste-Sudeste. Atualmente mantem-se instaladas e funcionantes 207 equipes (Lopes, 2007; Souza et. al., 2007; Brasil, 2010a).

O sofrimento psíquico, em suas diversas formas, constitui importante causa de perda de qualidade de vida na população em geral. A rea- 
lidade das ESF demonstra que, cotidianamente, deparam-se com problemas de "saúde mental", no entanto, são referidas dificuldades pelas respectivas, para lidar com o sofrimento psíquico, incluindo especialmente a abordagem das questões psicossociais e o manejo de transtornos mentais (Souza et. al., 2007; Brasil, 2009b).

Desta forma, remete-se a uma revisão crítica das concepções relacionadas aos processos de saúde-doença e da formação profissional, pois a construção da saúde, como processo social, requer novos dispositivos em saúde e um novo perfil profissional, mais sensível para perceber a real necessidade da população $(\mathrm{Ca}-$ valcante, 2007).

Mostrando-se a estratégia SF potencial catalisador da Reforma Psiquiátrica, tendo o Enfermeiro como membro desta e "ser cuidador", objetivou-se identificar e analisar a percepção do enfermeiro da atenção básica/equipe de saúde da família frente o cuidado ao portador de transtorno mental, uma vez que as atuais políticas voltadas à atenção em saúde mental buscam reabilitar e reinserir o portador de transtorno mental junto ao "seio" de sua família e comunidade.

\section{TRAJETÓRIA METODOLÓGICA}

Trata-se de uma pesquisa de caráter descritivo, exploratório com abordagem qualitativa (Gil, 2007; Minayo, 2008; Lakatos; Marconi, 2010). Foram realizadas entrevistas semi-estruturadas com 8 enfermeiros que atuam nas equipes de saúde da família localizadas na Região Centro/Norte de Teresina, a qual capital do estado do Piauí, Brasil, que aceitaram participar da pesquisa. Destacamos que esta é uma região que concentra os bairros mais populosos e pessoas com menor poder aquisitivo e abrange a maior parte dos serviços de saúde mental (Lopes, 2007).
Os dados foram produzidos através de entrevistas com base em um roteiro semiestruturado, de acordo com Minayo (2008), composto de duas partes: a primeira caracterização do sujeito e a segunda contendo uma pergunta aberta relacionada ao cuidado prestado pelo enfermeiro ao portador de transtorno mental. Realizadas individualmente e gravadas em dispositivo eletrônico (Mp3), posteriormente, transcritos e numerados.

Os dados foram analisados conforme análise temática segundo Minayo (2008), buscado a presença evidente da essencialidade da identidade do entrevistado. Obedecendo, assim, os critérios do ponto de saturação.

A pesquisa antes de ser realizada foi submetida à Comissão de Ética da Fundação Municipal de Saúde (FMS) de Teresina/PI; aprovado em 27 de fevereiro de 2012, sob MEMO CAA/N ${ }^{\circ}$ 62/2012, e ao Comitê de Ética em Pesquisa da Faculdade de Saúde, Ciências Humanas e Tecnológicas do Piauí - NOVAFAPI, instância colegiada, constituída pela IES em respeito às normas da Resolução no 196 , de 10 de outubro de 1996, do Conselho Nacional de Saúde - CNS, sob CAAE: 0472.0.043.00011. O CEP apresenta caráter multidisciplinar e transdisciplinar, incluindo a participação de profissionais da área de saúde, exatas e humanas, além de um representante do corpo administrativo da IES.

\section{RESULTADOS E DISCUSSÕES}

Os depoimentos foram coletados a partir de visitas às ESF em horário de funcionamento, de segunda a sexta-feira, manhã ou tarde. E ainda, conforme disponibilidade dos sujeitos da pesquisa.

Os dados foram analisados e agrupados em três categorias distintas: Estigma relacionado ao Portador de Transtorno Mental; Ações de 


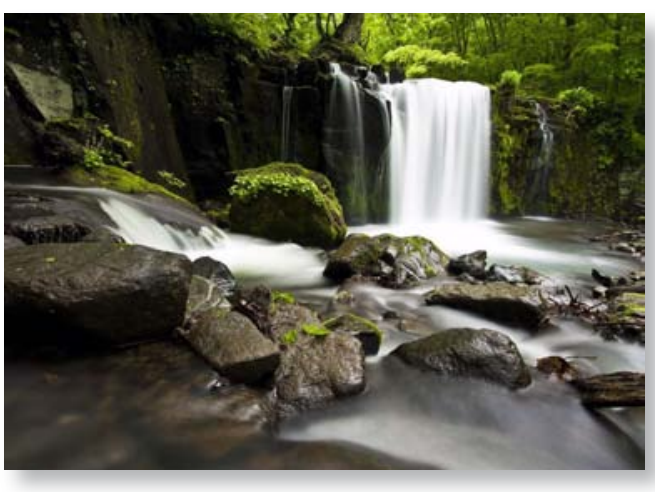

cuidado ao Portador de Transtorno Mental na Atenção Básica; Necessidade de articulação dos serviços.

\section{Estigma relacionado ao Portador de Trans- torno Mental}

A promoção da Saúde Mental e a luta pela desospitalização passam, hoje, pela efetiva implantação de uma rede comunitária que previna as situações de crise e ofereça alternativas à internação hospitalar. Por outro lado tenta-se romper com o paradigma da estigmatização que persiste atrelado as formas de cuidado ao portador de transtorno mental. Diante dessa notável situação, observou-se nas falas de alguns depoentes características notórias do estigma.

Nós levamos uma paciente com transtorno mental para as nossas atividades, [...] entrou na piscina após o almoço e fez coco [...]. Eu não vou tentar levar um portador de transtorno mental pra um passeio e lá não deixá-lo 'banhar' de piscina, porque corre o risco dele evacuar [...] Então é o que eu tô dizendo, tem algumas coisas que são utopia, você ta envolvendo (referindo-se ao portador de transtorno mental nas ações desenvolvidas pela ESF), né?! [...]. (Ent. 07)
Para Ferreira (2000), estigmatizar significa: marcar com ferrete; condenar; censurar. O estigma e a discriminação incluem preconceito, estereótipos, medo, embaraço, raiva e rejeição, violação de direitos básicos do ser humano e da liberdade, menores oportunidades de educação e perda dos direitos civis, políticos, econômicos, sociais e culturais (Brasil, 2008a).

A enfermagem, bem como todos os membros integrantes das equipes da estratégia SF são tidos como habilitados a assumir o tratamento dos transtornos mentais mais comuns, como por exemplo: quadros depressivos e ansiosos, e até psicoses mais simples. São também, capazes de detectar novos casos, identificar, intervir e prevenir fatores de risco, garantindo, assim, a continuidade e o efetivo impacto das ações de desospitalização e reintegração social dos pacientes portadores de sofrimento psíquico, bem como o fim do estigma da doença mental (Villela; Scatena, 2004).

Os profissionais de saúde, sob essa perspectiva, passam a potencializar suas capacidades, a mobilizar recursos e a assumir que os resultados dessa nova proposta na esfera da Saúde Mental devem ser baseados no cuidado inserido no campo coletivo dos indivíduos, e não mais somente no campo individual (Machado, 2010).

\section{Ações de cuidado ao Portador de Transtorno Mental na Atenção Básica}

A Saúde da Família apresenta-se como estratégia do cuidado que valoriza os princípios de formação de vínculo com a população, a integralidade na atenção e trabalho em equipe. Apontando, assim, para a necessidade de inserir questões de saúde mental em sua dinâmica diária de práticas de saúde, uma vez que a chegada de usuários com sofrimento psíquico nas unidades de saúde é bastante freqüente, as 
queixas são as mais variadas e para isso seria importante a equipe esteja preparada para oferecer maior resolutividade aos problemas destes usuários.

Nas entrevistas a seguir, pode-se observar que os enfermeiros pesquisados apontam para déficits diversos, principalmente a falta de conhecimento na área, estabelecendo uma lacuna no processo de cuidado.

[...] Eu acho muito complicado, acho complexo, eu não tenho muita afinidade, [...] não me sinto preparada para atuar em cima, fazer uma busca ativa e tentar resolver o problema dessas pessoas[...]. (Ent. 03)

Olhe essa coisa de cuidado ao portador de transtorno mental ela tá muito teórica, ainda no momento. [...] não dá tempo dar uma assistência ao paciente portador de transtorno mental com o tanto de coisa que o enfermeiro da estratégia Saúde da Família tem pra fazer, [...]. Então assim, não dá, é utopia, é utopia. (Ent. 07)

Percebe-se que o cuidado em saúde mental é deixado de lado sob diversos pretextos, dentre estes, a falta de capacitação na área, a ausência de afinidade, mas será que estes são realmente motivos que justifiquem a ausência do desenvolvimento dessas atividades? Cada ação desenvolvida pela equipe de saúde da família deve se reportar aos princípios que norteiam o próprio SUS, dentre estes, a universalidade que diz que todos os pacientes têm o direito a este atendimento.

O Manual de Enfermagem (PSF) do Ministério da Saúde (2002b) chama a atenção, não só do enfermeiro, mas de toda a equipe de saúde, para que percebam a importância da multicausalidade dos agravos à saúde.

Evidencia-se na fala abaixo uma aproximação dessa visão que contradiz os discursos anteriormente colocados, buscando afastar-se do pensamento de cuidado diferenciado e aproximar-se do público em questão mediante ações de cuidado desenvolvidas no seu dia-a-dia.

[...] quando o paciente ele está estabilizado, usando a medicação normalmente, então a gente insere esse paciente dentro do próprio cronograma de ação da unidade. [...] a gente não vê apenas o transtorno, a gente vê o paciente como um todo, [...] claro respeitando a limitação de cada um. (Ent. 06)

O Ministério da Saúde reconhece como fundamental para transformar a assistência a formação do profissional de saúde sob a ótica da ação integral, acesso universal, equidade, participação e controle social (Spandini; Souza, 2010).

As intervenções de enfermagem em saúde mental e psiquiátrica abrangem um amplo domínio, não só de locais de atuação como de clientela, ou seja, no lar, na escola e em serviços que exigem a mais especializada assistência ao ser humano. Cujo ponto crucial são as relações interpessoais desenvolvidas entre cliente, familiares e profissionais da área da saúde (Stefanelli; Fukuda; Arantes, 2011).

As percepções e as práticas de promoção da saúde, aqui expostas, voltadas ao portador de transtorno mental na Atenção Básica, apontam para a ausência de conhecimento nesse campo, emergindo, assim, o pensamento de que todo cuidado vincula-se com habilidades e conhecimento de público à trabalhar. Resgatamos aqui os conceitos de Boff (1999, p. 34) 
e Waldow (2004) que dizem: o cuidado é um processo, que se acha a priori, antes de toda atitude e situação do ser humano, um modo de se relacionar com alguém que envolve desenvolvimento e cresce em confiança mútua.

\section{Necessidade de articulação dos serviços}

As transformações no contexto da assistência psiquiátrica nos últimos anos têm trazido importantes contribuições para repensar o cuidado psiquiátrico no contexto brasileiro. Com a reforma psiquiátrica, procura-se desconcentrar o olhar sobre a doença, para dar ênfase ao sujeito. Nesse sentido a reforma psiquiátrica propõe o nascimento de novos dispositivos de tratamento, substitutivos do manicômio e inseridos na comunidade onde vive o sujeito. No contexto brasileiro, o Centro de Atenção Psicossocial.

Assim, esse deve estar articulado em parceria com outros serviços de saúde, para que possa dar conta de contemplar as diferentes dimensões da vida humana que vivencia uma situação de sofrimento mental. A pesquisa permitiu identificar que os enfermeiros fazem poucos atendimentos aos portadores de transtornos mentais. E quando o fazem, suas ações se destinam a encaminhamento médico ou aos CAPS, por exemplo. Estabelecendo um cuidado, muitas vezes, apenas paliativo. Consideram que o CAPS, assim como outras instituições de cuidado em saúde mental é que tem realmente a verdadeira responsabilidade pelo acolhimento e cuidado desse paciente. Entendem como dificuldade a impossibilidade de haver cuidado continuado pela não existência de articulação entre referencia e contra-referência.

[...] as equipes da Saúde da Família, têm dificuldade [...] encaminham, mas não sabem se foi recebido lá. [...] O fato é que, um CAPS pra dar conta de toda a região norte, [...] é realmente complicado, [...] por outro lado, tem que dar conta, e ai fica nesse impasse. (Ent. 04)

A referência é outra coisa difícil, [...]. Contra-referência não existe, [...], a gente já tentou encaminha pra o CAPS. Ai depois: Há a Assistente Social vai visitar, vai providenciar, [...] nunca houve nenhuma resposta pra gente. (Ent. 08)

No campo da saúde ainda vemos diferentes processos de trabalho centrados em uma lógica em que o modelo biomédico é hegemônico, a especialização parcela o conhecimento, as relações entre os trabalhadores são pautadas na verticalização e na hierarquia institucional e o cuidado é fragmentado, com poucas reflexões sobre o cuidado totalizador da vida. Nesse caso, a saúde e o trabalhador vão se alienando, distanciando-se, deixando de lado a valorização, a autonomia e a libertação de sujeitos (Pinho; Hernández; Kantorski, 2010).

A pouca afinidade das equipes com os conceitos de saúde e doença mental distancia a unidade das reais necessidades da população nesse campo, e a atitude mais freqüente é o encaminhamento para os serviços especializados. Tal condição ocasiona a criação involuntária de um circuito de medicalização excessiva. Essa situação, por sua vez, é corroborada com as dificuldades gerenciais e de investimento do poder público, numa conjugação que dificulta e estimula abaixa resolutividade (Barban; Oliveira, 2007).

Os Centros de Atenção Psicossocial (CAPS), entre todos os dispositivos de atenção à saúde mental, têm valor estratégico para a Reforma Psiquiátrica brasileira. Cabendo-lhe o acolhimento e a atenção às pessoas com 
transtornos mentais graves e persistentes, procurando preservar e fortalecer os laços sociais do usuário em seu território (Brasil, 2005; Brasil, 2007b; Brasil, 2011a).

A construção de uma rede comunitária de cuidados é fundamental para a consolidação da Reforma Psiquiátrica. Uma rede se conforma na medida em que são permanentemente articuladas outras instituições, associações, cooperativas e variados espaços das cidades. É portanto fundamento para a construção desta rede a presença de um movimento permanente, em busca da emancipação das pessoas com transtornos mentais (Brasil, 2005).

Permeando esse cenário, diante de todas as dificuldades expostas, há quem consiga notar uma luz no fim do túnel. Isso pode ser percebido nas falas seguintes:

O que ta faltando realmente são ações de fortalecimento [...], não só da Estratégia, quanto da ação do CAPS. O que não deixa de ser tudo atenção básica [...], ela precisa ser melhor planejada e executada. (Ent. 04)

[...] paciente está sendo acompanhado pela gente dentro da estratégia Saúde da Família, mas qualquer alteração a gente retorna ele pra o CAPS, [...], conseguimos fazer essa parceria, [...]. Graças a Deus, muito bem compartilhada, na realidade, muito bem aceita e nos ajuda muito [...]. (Ent. 06)

Para o Ministério da Saúde (2005) é fundamental que somente uma organização em rede, e não apenas um serviço ou equipamento, é capaz de fazer face à complexidade das demandas de inclusão de pessoas secularmente estigmatizadas, em um país de acentuadas

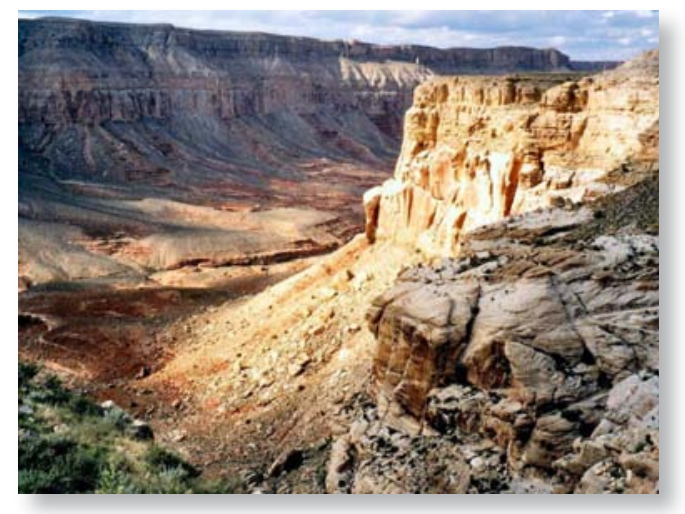

desigualdades sociais. Para a organização desta rede, a noção de território é especialmente orientadora.

O território é a designação não apenas de uma área geográfica, mas das pessoas, das instituições, das redes e dos cenários nos quais se dão a vida comunitária. Trabalhar no território significa assim resgatar todos os saberes e potencialidades dos recursos da comunidade, construindo coletivamente as soluções, a multiplicidade de trocas entre as pessoas e os cuidados em saúde mental (Brasil, 2005; Brasil, 2011a).

Nesse sentido, Passos (2007), aponta que não há, efetivamente, como fazer saúde pública centrada no especialista. Certamente, superá-lo não é tarefa isoladamente de uma pessoa ou de uma especialidade em particular, mas da coletividade humana. Dessa forma, cabe aos profissionais que atuam nas ESF a busca pelo conhecimento do ser generalista, que atua no acompanhamento de problemas de saúde que abrangem toda a comunidade e, dentre estes, o transtorno mental se insere como problema evidente e gritante para a vida em sociedade.

\section{CONSIDERAÇÕES FINAIS}

Com este trabalho buscamos identificar a percepção do Enfermeiro da atenção básica/ ESF frente ao cuidado do portador de transtorno mental, objetivando analisar os aspectos 
favoráveis e desfavoráveis frente estas ações. Mostrando-se, ainda, de grande relevância, uma vez que, encontramos poucos estudos no campo, principalmente por parte da enfermagem. Identificamos ainda que, no município de Teresina, capital piauiense não foram encontrados estudos com esse tipo de abordagem, revelando a urgência de produção científica na área e abordagem.

O processo da Reforma Psiquiátrica brasileira vem galgando a duras penas, numa tentativa de desmistificar que o lugar do "louco" é com "louco", e não na comunidade. Se ao Enfermeiro cabe atribuições básicas de execução de ações básicas de assistência e promoção da qualidade de vida, também, não deve esquecer que quem porta transtorno mental, seja em que nível for, sem dúvidas ocupa o mesmo espaço das demais pessoas.

Neste momento é necessário delinear o papel de cada sujeito integrante da sociedade civil: gestores, profissionais de saúde e comunidade. Quem sabe do ponto em que se deve levar em conta que existe um componente de sofrimento subjetivo associado a toda e qualquer doença. Buscando manter uma cultura de cuidados que incorpore novos conceitos pertencentes ao adoecimento mental, ao invés de esperar que o acometido mentalmente procure o serviço de saúde. Uma vez que, pode-se dizer que todo problema de saúde é também mental, e que toda saúde mental é também produção de saúde.

\section{REFERÊNCIAS}

- Amarante, P. (2007) Saúde mental e atenção psicossocial. 2. ed. Editora Fiocruz, Rio de Janeiro:

- Barban, E. G; Oliveira, A. A. (2007) O modelo de assistência da equipe matricial de saúde mental no programa saúde da família do município de São José do Rio Preto (Capacitação e educação permanente aos profissionais de saúde na atenção básica). Disponível em: http://www. cienciasdasaude.famerp.br/racs_ol/vol-14-1/ID224.pdf. Acesso em: 05 jun. 2012.

- Boff, L. (1999) Saber cuidar: ética do humano - compaixão pela terra. 10. ed. Petrópolis, Vozes.

- Brasil. (2002) Ministério da Saúde. Estabelecer que os Centros de Atenção Psicossocial poderão constituir-se nas seguintes modalidades de serviços: CAPS I, CAPS II e CAPS III. Portaria GM n. 336, de 19 de fevereiro de 2002. Lex: Presidência da República - Casa Civil: Subchefia para Assuntos Jurídicos Brasília. Legislação Federal e marginalia.

- Brasil. (2002) Ministério da Saúde. Secretaria de Gestão do Trabalho e da Educação na Saúde.Profissionalização de auxiliares de enfermagem: cadernos do aluno saúde mental. Brasília.

- Brasil. (2003) Ministério da Saúde. Secretaria de atenção à saúde. Saúde mental e atenção básica: o vínculo e o diálogo necessários: Inclusão das ações de saúde mental na atenção básica. Brasília.

- Brasil. (2005) Ministério da Saúde. Secretaria de Atenção à Saúde. Coordenação Geral de Saúde Mental. Reforma psiquiátrica e política de saúde mental no Brasil: documento apresentado a Conferência Regional de Reforma dos Serviços de Saúde Mental: 15 anos depois de Caracas. OPAS. Brasília.

- Brasil. (2006) Ministério da Saúde. Aprova a Política Nacional de Atenção Básica, estabelecendo a revisão de diretrizes e normas para a organização da Atenção Básica para o Programa Saúde da Família (PSF) e o Programa Agente Comunitário de Saúde (PACS). Portaria GM n. 648, de 28 de março de 2006. Lex: Presidência da República - Casa Civil: Subchefia para Assuntos Jurídicos Brasília. Legislação Federal e marginalia.

- Brasil. (2007) Ministério da Saúde. Secretaria de Atenção à Saúde. Política Nacional de Atenção Básica. 4. ed. Brasília.

- Brasil. (2008) Agência Nacional de Saúde Suplementar. Diretrizes Assistenciais para a Saúde Mental na Saúde Suplementar. Rio de Janeiro: ANS.

- Brasil. (2009) Ministério da Saúde. Secretaria de Atenção à Saúde. Departamento de Atenção Básica. Cadernos de 
Atenção Básica: Diretrizes do NASF - Núcleo de Apoio a Saúde da Família. Brasília.

- Brasil. (2009) Secretaria de Estado de Saúde do Distrito Federal. Núcleo de Saúde Mental - Gerencia de Enfermagem. Cartilha de orientação em Saúde Mental: um caminho para a inclusão social. Brasília.

- Brasil. (2010) Ministério da Saúde. Coordenação Geral de Saúde Mental, Álcool e Outras Drogas. Saúde Mental em Dados - 7. Brasília. Disponível em: <www.saude.gov. br e www.saude.gov.br/bvs/saudemental>. Acesso em 29 set. 2011.

- Brasil. (2011) Ministério da Saúde. Secretaria de Atenção à Saúde. Saúde Mental no SUS: as novas fronteiras da Reforma Psiquiátrica. Relatório de Gestão 2007-2010. Brasília.

- Cavalcante, A. C. S. (2007) Desafios em promover saúde mental na atenção básica. Revista FACID 3(1): 203-217

- Chamma, R. et. al. (2011) Serviços de atendimento em Saúde Mental e psiquiatria. In: sefanelli, M. C.; fukuda, I. M. K.; arantes, E. C. Enfermagem psiquiátrica em suas dimensões assistenciais Manole, . Barueri :40-55.

- Ferreira, A. B. H. (2000) Miniaurélio Século XXI Escolar: O minidicionário da língua portuguesa. 4 ed. rev. ampliada. Nova Fronteira, Rio de Janeiro.

- Gama, J. R. de A. (2008) Um estudo histórico e conceitual sobre a clínica da reforma Psiquiátrica brasileira. 2008. 237 f. Tese de Doutorado, apresentado ao Instituto de Medicina Social da Universidade Estadual do Rio de Janeiro - Área de Concentração: Ciências Humanas e Saúde.

- Gil, A. C. (2007) Como elaborar projeto de pesquisa. 4. Atlas. São Paulo.

- Lakatos, E.M; Marconi, M.A. (2010) Fundamentos de metodologia cientifica. 7. ed. Atlas, São Paulo.

- Leal, B. M. M. L. (2010) Movimentos do cuidado em saúde mental na estratégia saúde da família. 2010. 149 f. Dissertação (Mestrado em Enfermagem Psiquiátrica) - Programa de Pós-graduação em Enfermagem Psiquiátrica, Escola de Enfermagem da Universidade de São Paulo, São Paulo. (Dissertação)

- Lopes, D. C. T.; Dimenstein, M. (2007) Mapeamento do Trabalho em Saúde Mental na Atenção Básica na Rede
SUS Teresina PI. In. XIV ENCONTRO NACIONAL DA ABRAPSO. Recife, 2007. Anais... Recife: ABRAPSO, 2007. Disponível em: <http://www.abrapso.org. br/siteprincipal/anexos/AnaisXIVENA/conteudo/pdf/ trab_completo_56.pdf>. Acesso em: 18 out. 2011.

- Machado, R. M. (2010)Transtornos Psiquiátricos: Uma abordagem epidemiológica do alcoolismo na região centro oeste de Minas Gerais. 2010. 180 f. Tese de Doutorado, apresentado à Escola de Enfermagem de Ribeirão Preto/USP - Área de Concentração: Enfermagem Psiquiátrica. (Tese)

- Mendes, M. H.; Freitas,V. A.; Gomes, E. T. (2009) Consulta de enfermagem:uma prática necessária aos indivíduos com transtornos mentais. Rev. Enferm. Integrada, Ipatinga: Unileste, v. 2, n. 1 Jul./Ago. (Artigo Revista)

- Minayo, M. C. de S. (2008) O Desafio do conhecimento: pesquisa qualitativa em saúde. 11. ed. São Paulo: Hucitec. (Livro)

- Moura, M. C.; Bernardes, S. B.; Rocha, M. L. F. (2010) A percepção dos enfermeiros de uma universidade pública do Piauí sobre saúde mental na estratégia saúde da família. Rev. Enferm. em Foco, Brasília, v. 1, n. 2, p. 66-68, ago.; 2010. (Artigo Revista)

- Passos, F. (2007) Saúde Mental Atenção Básica: Desafio para além e para aquém da contenda hospitalizar/desospitalizar. In: Rosa, L. C. S.; Bona, M. E. C. União em Saúde Mental. Teresina: EDUFPI, 2007. p. 103-129. (Livro)

- Pinho, L. B.; Hernández, A. M. B.; Kantorski, L. P. (2010) Trabalhadores em saúde mental: contradições e desafios no contexto da reforma psiquiátrica. Esc. Anna Nery. Rio de Janeiro, v. 14, n. 2, p. 260-267, abr./jun.; 2010. (Artigo Revista)

- Santos, A. M. C. C. (2008) "Gênero e Saúde Mental: a vivência de identidades femininas e masculinas e o sofrimento psíquico na sociedade brasileira contemporânea. Algumas reflexões a partir de relatos de pacientes diagnosticados como portadores de transtornos mentais severos do CAPS - Araraquara - SP.” 2008. 180 f. Dissertação (Mestrado em Sociologia) - Programa de Pós-graduação em Sociologia, Faculdade de Filosofia, Letras e Ciências Humanas da Universidade de São Paulo, São Paulo. (Dissertação) 


\section{Cultura de las Cuidados}

- Souza, A.J.F. et. al. (2007) A saúde mental no Programa de Saúde da Família. Rev. Bras. Enferm. Brasília, v. 60, n. 4, jul./ago.; 2007. (Artigo Revista)

- Spandini, L. S.; Souza, M. C. B. M. (2010) O preparo de enfermeiros que atuam em grupos na área de saúde mental e psiquiatria. Esc. Anna Nery. Rio de Janeiro, v.14 n.2, p. 355-360, abr./jun.; 2010. (Artigo Revista)

- Stefanelli, M. C. (2011) Enfermagem psiquiátrica no século XXI. In: Stefanelli, M. C.; Fukuda, I. M. K.; Arantes, E. C. Enfermagem psiquiátrica em suas dimensões assistenciais. Barueri: Manole, 2011. p. 3-15. (Livro)
- Stefanelli, M. C.; Fukuda, I. M. K.; Arantes, E. C. (2011)

Enfermagem psiquiátrica em suas dimensões assistenciais. Barueri: Manole. (Livro)

- Townsend, M.C. (2002 Enfermagem Psiquiátrica: conceitos de cuidado. 3.ed. Rio de Janeiro: Guanabara Koogan S.A, 2002. (Livro)

- Vilela, S. C.; Scatena, M. C. M. (2004) A enfermagem e o cuidar na área de saúde mental. Rev. Bras. Enferm. Brasilia, v. 57, n. 6, nov./dec.; 2004. (Artigo Revista)

- Waldow, V. R. (2004) O cuidado na saúde: as relações entre o eu, o outro e o cosmos. Petrópolis: Vozes. (Livro)

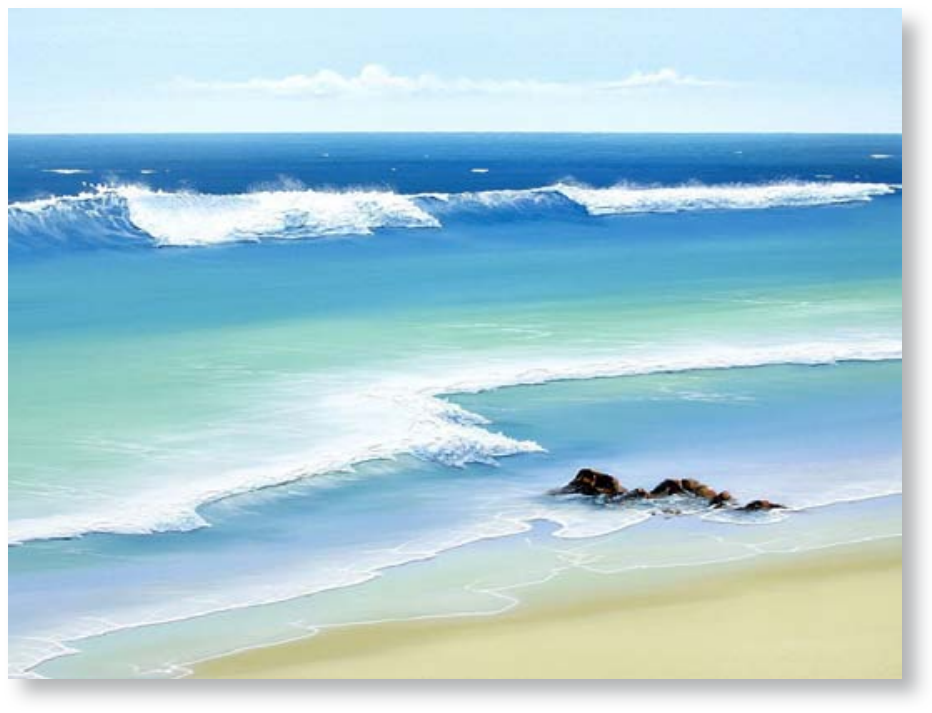

